\title{
Pratiques
}

Linguistique, littérature, didactique

\section{Influence de l'enseignement du lexique en familles de mots sur la constitution du lexique orthographique}

Véronique Rey, Leila Lions et Sandra Radmacher

\section{OpenEdition}

\section{Journals}

Édition électronique

URL : http://journals.openedition.org/pratiques/3496

DOI : $10.4000 /$ pratiques.3496

ISSN : 2425-2042

Éditeur

Centre de recherche sur les médiations (CREM)

\section{Édition imprimée}

Date de publication : 15 décembre 2012

Pagination : 169-181

Référence électronique

Véronique Rey, Leila Lions et Sandra Radmacher, « Influence de l'enseignement du lexique en familles de mots sur la constitution du lexique orthographique », Pratiques [En ligne], 155-156 | 2012, mis en ligne le 18 décembre 2017, consulté le 01 mai 2019. URL : http://journals.openedition.org/ pratiques/3496; DOI : 10.4000/pratiques.3496 


\title{
Influence de l'enseignement du lexique en familles de mots sur la constitution du lexique orthographique
}

\author{
Véronique Rey \\ Professeur des Universités, Linguiste, CNRS UMR 8562 \\ Leila Lions, Sandra Radmacher \\ Orthophonistes
}

\section{Introduction}

Les bases de notre réflexion, tout au long de cette étude, s'inspireront fortement de la théorie développée par Doneux (2001). Pour ce linguiste, lorsque l'on enseigne l'écriture d'une langue alphabétique comme le français, il est important de déterminer, avant toute chose, la manière dont la graphie rend compte des unités phonologiques car la sécurité d'inscription chez l'apprenant est liée aux conventions d'écriture des unités phoniques. Ainsi, il serait possible, toujours selon Doneux, de dégager deux systèmes d'écriture en français :

- une « écriture aléatoire » où l'inscription des unités phoniques de la langue n'aurait aucune fermeté (un phonème peut recevoir plusieurs graphies). L'écriture des mots est aléatoire et tributaire de la mémorisation de leur forme par le scripteur (exemple «thym »);

- une "écriture prédictible » où un phonème est toujours transcrit de la même façon. L'apprenant doit donc intégrer les règles de correspondance bi-univoques entre un phonème et un graphème. La prédictibilité d'inscription des mots sera donc entière et directe.

Cependant Doneux attire l'attention sur le fait que la référence phonématique n'est pas toujours un gage absolu d'écriture correcte (ex : /metse/ s'écrit « médecin », /tapi/ s'écrit « tapis »). En effet, certains éléments phoniques subissent des altérations (assimilation, dissimilation, harmonisation...). Le seul moyen alors de retrouver l'écriture correcte d'un mot qui aurait subi des altérations phonologiques est d'examiner un autre niveau : le niveau morphologique.

C'est par la manipulation, à l'oral, des morphèmes et des mots, dans des con- 
textes divers que le sujet peut induire notamment des unités qui sont présentes en structure sans toutefois être prononcées (ex : le «s » dans « tapis » apparaît en dérivation dans «tapisserie », « tapissier » et sera considéré comme un phonème structurel).

Doneux envisage, dans le cadre de l'enseignement de l'orthographe du français une combinaison entre plusieurs éléments :

- Les éléments prédictibles : ils peuvent être directes (phonologique) ou indirectes (morpho-phonologique)

- Les éléments aléatoires : ils sont tributaires de la mémorisation des formes par le scripteur.

La distinction dans l'écriture d'une langue entre ce qui est phonologiquement prédictible et ce qui est aléatoire aurait une pertinence dans les processus d'apprentissages de l'écriture de chaque catégorie de mots. Le propos de cette étude est d'appréhender expérimentalement la pertinence de cette distinction : un entraînement morphologique oral aurait-il un impact différent d'un entraînement strictement visuel sur les acquisitions orthographiques lexicales?

Afin d'explorer cette distinction, nous présenterons un cadre théorique comprenant d'une part l'orthographe du français (partie 1) et d'autre part le processus cognitif devant prendre en compte des modules linguistiques (phonologie et morphologie) suivant la nature du mot orthographié (partie 2). Enfin, une étude expérimentale (partie 3) permettra de tester l'impact d'un entraînement morphologique sur l'acquisition de l'orthographe et valider ou non l'approche de Doneux, dans le domaine de l'apprentissage.

\section{L'orthographe du français, une écriture morpho-phonologique}

L'orthographe est la forme normée d'écriture d'une langue donnée, régissant ainsi la manière d'écrire les mots isolés et les mots composant une phrase. Chaque orthographe, selon Catach, N. (1978, 1980), Frost \& Katz (1992), Fayol \& Jaffré (1997) et Jaffré (2003), est façonnée par un ensemble de facteurs linguistiques, historiques, socioculturels et constitue ainsi un héritage qui nécessite un long apprentissage. Traditionnellement, le système graphique français est présenté comme un système alphabétique mais ce dernier n'épuise pas, loin sans faut, le maniement de l'orthographe du français. Même si la transcription graphique par la correspondance des phonèmes en lettres ou graphèmes assure un premier enregistrement de la langue orale, la transcription graphique renvoie aussi à la construction des mots au moyen de flexions et de dérivations en se référant à l'étymologie, la morphologie et à la sémantique (Blanche-Benveniste \& Chervel (1969), Rieben et al. (1997), Jaffré, (2003). Les relations en langue française entre l'oral et l'écrit en deviennent complexes et parfois aléatoires comme le souligne Doneux (2001). Le français ne s'écrit donc pas toujours comme il se prononce et ceci dès l'observation du nombre de lettres au regard du nombre de phonèmes : il y aurait 40 phonèmes en français et 26 lettres (Jaffré, 2003).

Par delà l'écriture des phonèmes, l'écriture des mots est difficile car le fran- 
çais est une langue flexionnelle et dérivationnelle. La variation des mots selon le genre, le nombre, la personne, le temps et le mode au sein d'une phrase est un phénomène qui relève de la flexion et, en langue écrite, de l'orthographe grammaticale. L'analyse de la phrase en constituants (syntagme nominal, syntagme verbal) est une capacité nécessaire pour automatiser progressivement cette orthographe grammaticale (Anderson, 2000). La transformation des mots en fonction du sens relève du domaine du lexique et concerne la dérivation lexicale (par exemple, le fil, la file, défiler, refiler, enfiler). Les variations dérivationnelles ont pour effet mécanique d'accroître les ressources du lexique (Rey \& Gala (2011). Catach (1978), Doneux (2001) et d'autres ont attiré 1'attention sur ces lettres qui comportent une information sur le radical. C'est ainsi que le mot chat est présent à l'intérieur de plusieurs lexèmes de la famille « chat, chaton, chatte, chatière » ou que le mot « pot» se rencontre dans la série suivante : « dépotage, dépotement, dépoter, dépotoir, empoter, pot, potage, potager, poterie, potière, rempotage, rempoter ». Dans les deux cas, la lettre « $t$ » finale des mots « chat» et «pot» est motivée non par la sortie phonique immédiate mais par une sortie phonique différée en situation de dérivation lexicale. Les choses se compliquent en abordant les voyelles. Les graphèmes « un » ou « in » pour transcrire les voyelles nasales seront choisis souvent en fonction d'un choix lexical en dérivation. Les mots « cousin » et « cousine » ont un « $i$ » en commun, car ce dernier correspond au phonème /i/ du mot/kuzind/; les mots « brun » et « brune», sur ce même principe, ont un « u » en commun; et « européen » s'écrit avec le graphème « en » car le mot associé est « européenne »; la lettre «e » est alors commune aux deux mots. Les mots comme «paix, pacification; urbain, urbaniser, urbaniste ; vain, vaine, vanité » présentent tous la lettre «a » en commun, comme un lien graphique entre les mots d'une famille lexicale car le phonème /a/s'entend dans les mots en dérivation. De même, dans le cas du mot « château », le graphème " eau » est motivé par le fait que le phonème [œ] («e ») existe oralement dans les mots dérivés comme « châtelain » (il en est de même pour beaucoup de mot se terminant en « eau » (Doneux, 2001).

L'orthographe du français repose donc non seulement sur un principe alphabétique mais aussi sur un principe morpho-phonologique car il est nécessaire tant au niveau grammatical que lexical, d'introduire les propriétés morphologiques des mots pour rendre compte de leur écriture. Il y a complémentarité entre plusieurs principes :

- Lorsque le niveau phonologique sert de référent, la prédictibilité est toujours directe : ce qui est entendu comme /b/ s'écrira toujours «b » en français.

- Si le référent est structurel, la prédictibilité est indirecte : elle est soumise à une condition qui se vérifie par des manipulations morphologiques à l'oral.

- Enfin, il y aurait un niveau aléatoire où ni la sortie phonologique ni la sortie morpho-phonologique n'assure l'orthographe du mot : seule la mémorisation visuelle permet de l'orthographier correctement. Cela concerne des mots comme «thym, puzzle, poids...».

Ces différents principes relèvent alors de processus cognitifs hétérogènes. Cela nous conduit au point suivant. 


\section{Acquisition de l'orthographe lexicale : un processus cognitif}

Depuis les observations cliniques auprès des adultes présentant des dysorthographies suite à des perturbations neurologiques (Coltheart, 1978), il s'avère que l'orthographe lexicale en lecture et en écriture repose sur une double procédure : la voie par assemblage et la voie par adressage. En effet, le lecteur-scripteur a deux possibilités : une selon laquelle les mots familiers sont traités directement par une reconnaissance visuelle globale. C'est la voie par adressage dite également voie lexicale. Une autre possibilité est de décomposer/recomposer le mot non familier en unités phonologiques. C'est la voie par assemblage, ou voie phonologique. La rapidité d'activation du stock lexical et les comparaisons simultanées avec plusieurs représentations proches sont proportionnelles à la fréquence d'usage des mots identifiés.

Cette première approche a le mérite de démontrer l'activité cognitive sous-jacente au maniement orthographique des mots mais demeure silencieuse sur la dimension morphologique des mots complexes. Différentes théories rendent compte de l'acquisition de la structure morphémique des mots chez l'enfant et de la capacité de ce dernier à manipuler explicitement cette structure (Frost \& Katz 1992). L'apprentissage de l'écrit et plus particulièrement la mise en place de l'orthographe lexicale pourrait reposer sur l'organisation du lexique orthographique. Ce dernier serait à l'interface des activités de lecture et d'écriture (Morais \& Robillard, 1998). Dans le cadre du modèle de Seymour (1997), la morphologie lexicale aurait un rôle dans la constitution de ce lexique orthographique. Seymour a proposé un modèle à double fondation concernant l'apprentissage de l'écriture, de la lecture et de l'orthographe. Selon lui, la phonologie et la morphologie interviennent au sein du processus d'apprentissage de l'orthographe. Cinq modules composent ce modèle : le processus logographique, le processus alphabétique, la conscience linguistique (phonologique et morphologique), la structure orthographique et la structure morphographique. Si ces cinq structures sont en lien constant, la structure orthographique et la structure morphologique jouent un rôle central.

Au sein de la morphologie lexicale, nous nous intéressons plus particulièrement aux familles de mots en dérivation. Dans de très nombreux cas en français, les mots d'une même famille partagent un radical graphique commun. Par exemple, les lettres muettes en position finale sont communes à plusieurs lexèmes de la même famille de mot (grand, grandeur, agrandir ; long, longueur, longer ; tapis, tapissier, tapisserie...). En lecture, il a été démontré que les lecteurs experts décomposent les mots morphologiquement complexes de faible fréquence (Carlisle 1988) et s'appuient sur la structure morphologique des mots pour améliorer leur accès lexical (Senechal \& Kearnan 2007). En écriture, si les travaux sur l'acquisition de l'orthographe grammaticale sont très nombreux, il n'en est pas de même pour l'orthographe lexicale (Sprenger-Charolles \& Casalis, 1996, Bourassa \& Treiman, 2000, Baumard, 2004).

Cette recherche s'inscrit uniquement dans le domaine de l'orthographe lexicale. En effet, au lieu de présenter les mots comme des unités isolées dont il faudrait apprendre l'orthographe visuellement, le propos est d'évaluer la pertinence de travailler le lien entre la conscience des familles de mots, à l'oral, et l'orthographe de ces mots. 


\section{3. Évaluation d'un entrânement morphologique sur l'acquisition de l'orthographe}

\subsection{Méthodologie}

Nous avons constitué deux groupes de 80 enfants : un groupe d'enfants « contrôles » (20 enfants de $6^{\mathrm{e}}$; la moyenne d'âge est de 11 ans 7 mois et 20 enfants de $5^{\mathrm{e}}$, la moyenne d'âge est de 12 ans 1 mois) ont appris l'orthographe des mots de façon isolée et un groupe d'enfants entraînés ( 20 enfants de $6^{\mathrm{e}}$, la moyenne d'âge est de 11 ans 6 mois et 20 enfants de $5^{\mathrm{e}}$, la moyenne d'âge est de 12 ans 9 mois) ont réalisé des exercices portant sur les familles lexicales à l'aide d'activités spécifiques. Les classes de $6^{\mathrm{e}}$ et de $5^{\mathrm{e}}$ ont été choisies en raison de leur situation charnière à leur arrivée au collège. L'ensemble des enfants a été évalué par des orthophonistes, en dictée de mots au début de l'entraînement, à la fin de l'entraînement, et un mois après l'entraînement. L'ensemble des entraînements s'est réalisé par groupe de 4 enfants avec les enseignants de français et par une orthophoniste, chargée du protocole. Ce dernier se déroule en quatre temps :

- Une dictée de 22 mots avant l'entraînement permet d'évaluer les connaissances lexicales des enfants (E1)

- Un entraînement basé sur le maniement de familles de mots à partir de jeux, entraînement que l'on nommera " entraînement morpho-phonologique lexical»

- Une dictée d'évaluation E2, des mêmes mots qu'en E1, quinze jours après le dernier l'entraînement

- Une dictée d'évaluation E3, six semaines après la dictée E2, afin de savoir si les acquisitions orthographiques se maintenaient ou non

\subsection{Matériel}

\subsubsection{Le choix des mots}

Le choix des mots répond à la demande des programmes de l'enseignement du français au collège (Bulletin Officiel spécial $\mathrm{n}^{\circ} 6$ du 28 août 2008) qui recommandent en sixième de travailler les domaines lexicaux des émotions et des religions et les genres littéraires (contes, récits) et en cinquième, il est préconisé d'aborder les domaines lexicaux des sensations, valeurs (références à une société ou à une période découverte à travers les œuvres étudiées en classe) et des genres littéraires (roman, comédie). A partir de ce cadre, les mots choisis ont été testés avec les bases de données lexicales Manulex (base de données lexicales qui fournit les fréquences des occurrences à partir d'un corpus de 54 manuels scolaires de classes en primaire - Lété, Sprenger-Charolles \& Colé, 2004) et Eole (échelle d'acquisition en orthographe lexicale de niveau scolaire élémentaire, testée auprès de 2000 classes - Pothier, 2004). A partir de la base POLYMOTS ${ }^{(1)}$, nous avons extrait des mots dont le radical est un mot autonome (ex : table) et des

(1) http://polymots.lif.univ-mrs.fr/v1/index.php 
mots dont le radical n'est pas un mot autonome (ex : cid dans accident). Des substantifs, des verbes et des adjectifs ont été retenus mais ne sont pas traités comme des variables dans notre protocole. Le tableau ci-dessous présente l'ensemble de la famille de mots, donné par ordre alphabétique, le mot souligné indique le mot de base de la famille, le premier pourcentage est celui de la base de données EOLE et le deuxième pourcentage est celui de MANULEX (l'indice de fréquence standard a été retenu). Deux mots, béat et blasphème ne sont pas recensés dans les deux bases de données mais ont été maintenus car ils relèvent des thématiques des programmes scolaires.

\begin{tabular}{|c|c|c|}
\hline MOTS & EOLE & MANULEX \\
\hline Béat, béate, béatement, béatifier, béatitude & - & - \\
\hline Bizarre, bizarrement, bizarrerie, bizarroïde & $44 \%$ & $58,1 \%$ \\
\hline Blasphème, blasphémateur, blasphématoire, blasphémer & - & - \\
\hline Cadence, cadencer, décadence, décadent & $44 \%$ & $43,1 \%$ \\
\hline Conte, conter, conteur, racontar, raconter & $79 \%$ & $60,8 \%$ \\
\hline Divin, dieu, divinement, diviniser, divinité & $78 \%$ & $19,3 \%$ \\
\hline Destin, destinataire, destination, prédestiner & $90 \%$ & $47 \%$ \\
\hline Fan, fanatique, profanation, profaner & $64 \%$ & $34,6 \%$ \\
\hline$\underline{\text { Las, }}$ délasser, inlassablement, lasse, lassitude & $30 \%$ & $41,6 \%$ \\
\hline Nerf, énerver, nerveux, nervure & $41 \%$ & $19,2 \%$ \\
\hline Ennui, nuis, ennuyeux, nuire, nuisance & $34 \%$ & $51,2 \%$ \\
\hline Orange, orangeade, oranger, orangeraie & $100 \%$ & $54,7 \%$ \\
\hline Orgueil, enorgueillir, orgueilleusement, orgueilleux & $10 \%$ & $49,5 \%$ \\
\hline Rite, ritualiser, rituel, rituellement & $81 \%$ & $33,3 \%$ \\
\hline Strophe, apostropher, catastrophe, catastrophique & $77 \%$ & $44,7 \%$ \\
\hline Temple, contemplatif, contemplation, contempler & $91 \%$ & $42,2 \%$ \\
\hline Angoisse, angoisser, anxiété, anxieux & $50 \%$ & $50,8 \%$ \\
\hline Ferveur, effervescence, effervescente, fervent & $67 \%$ & $21,6 \%$ \\
\hline Pitié, apitoyer, impitoyable, pitance & $78 \%$ & $53,6 \%$ \\
\hline Pudeur, pudibond, pudique, pudiquement & $71 \%$ & $24,3 \%$ \\
\hline
\end{tabular}

Tableau 1 : Familles de mots extraits de POLYMOTS

\subsubsection{Entraînements sous forme de jeux}

L'ensemble des entraînements s'est réalisé dans le cadre de la classe, sous la responsabilité de l'enseignante. Six jeux ont été construits pour entraîner les enfants.

Les mots cigognes : la base et les dérivés sont écrits sous forme de grilles. 
L'indiçage correspond à la visibilité des particularités orthographiques des affixes (préfixe ou suffixe). Les documents sont composés d'une feuille par familles de mots. Ces dernières sont proposées par ordre alphabétique. Les élèves ont un accès visuel du mot et doivent compléter les cases manquantes pour découvrir les autres mots de la même famille. L'évocation lexicale devrait permettre la découverte lexicale des suffixes.

Les mots croisés (Annexe 1) : Il y a des mots croisés pour chaque famille. Le principe est le même que les mots croisés ordinaires : les enfants travaillent sur la base d'une définition qui leur donne un accès sémantique au mot qu'ils doivent trouver. Les définitions sont extraites du dictionnaire Le Robert Junior, langue française poche (2004) et du dictionnaire Le nouveau Littré (2006), car certaines mots n'apparaissent pas dans Le Robert Junior.

Les mots en escalier (Annexe 2) : Sur une feuille, des grilles horizontales de longueur croissante évoque la forme d'un escalier. Sur ces grilles figurent la base ainsi que les lettres indices en fin de mots. L'élève doit compléter les cases vides. A chaque famille correspond une feuille.

Les syllabes mélangées : Ce sont des étiquettes manipulables et plastifiées. Chaque étiquette représente une syllabe appartenant à un des mots d'une famille étudiée. La base apparaît en caractère gras. Chaque enfant possède une famille et doit reconstituer l'ensemble. Cet exercice sollicite l'exploration visuo-spatiale, la représentation mentale, la mémoire sémantique et la mémoire de travail verbal.

Uno des mots : Il s'agit d'une adaptation du jeu du UNO qui invite les joueurs à se défausser de leurs cartes en associant la carte présente au centre du jeu soit sur le critère de famille soit sur le critère de couleur.

Version couleur : le jeu est présenté sous forme d'un ensemble de cinq familles. A une famille correspond une couleur. La couleur est une aide précieuse pour participer au jeu.

Version famille : le critère dominant est le mot ; plusieurs familles peuvent avoir la même couleur. Il y a donc une stimulation de lecture rapide et de reconnaissance visuelle du même radical.

Le radical en satellite : Au centre d'une feuille de papier apparaît un mot au départ duquel des flèches indiquent des mots à découvrir dont la première syllabe est donnée. Le but est de permettre aux élèves de trouver les noms appartenant à cette famille.

Les enfants témoins n'ont eu aucun de ces entraînements.

\subsection{Entraînement}

Les élèves sont répartis en groupes de quatre au sein de leur classe lors de l'entraînement. Ces groupes restent les mêmes durant toutes les séances.

Les exercices sont réalisés pendant 6 semaines au rythme de deux fois par semaine, pendant une heure. Chaque exercice aborde les 20 familles de mots; tous les exercices ne sont pas réalisés en une seule séance.

Les enfants contrôles ont appris par cœur les 20 mots de la dictée, sur une durée de 15 jours (10 mots par semaine). 


\subsection{Résultats}

Les résultats sont présentés en pourcentage pour une meilleure visibilité des données.

\subsubsection{Approche globale}

Le graphique de la figure 1 (page suivante) représente, de gauche à droite, les trois évaluations des enfants de la classe de $6^{\mathrm{e}}$ entraînés $\left(6^{\mathrm{e}} \mathrm{e}\right)$ et contrôles $\left(6^{\mathrm{e}} \mathrm{c}\right)$ et les trois évaluations des enfants de la classe de $5^{\mathrm{e}}$ entraînés $\left(5^{\mathrm{e}}\right.$ e) et contrôles $\left(5^{\mathrm{e}} \mathrm{c}\right)$.

Comme l'indique la figure 1, les enfants en E1 avaient globalement les mêmes compétences (colonnes $1,4,7 \& 10$ ) ; les enfants de $5^{\mathrm{e}}$ ont des performances meilleures que les enfants de $6^{\mathrm{e}}$.

Après l'entraînement, les résultats montrent la pertinence de l'entraînement par familles de mots, non seulement juste après l'entraînement (E2, colonnes 2 \& 8), mais également un mois après (E3, colonnes $3 \& 9$ ), à la différence de l'entraînement par mots isolés qui a un effet immédiat, mais qui ne se maintient pas (E3, colonnes $6 \& 12$ ). Ceci atteste que la transmission du lexique dans son organisation morphologique (en familles de mots) a un effet mnésique et structurant pour la mise en place du lexique orthographique. Selon le cadre théorique de Doneux (2001) \& Seymour (1997), l'appropriation de l'écriture repose sur la conscience linguistique comprenant la conscience morphologique (dont la manipulation en familles de mots) et la conscience phonologique. L'entraînement aurait donc un impact sur l'acquisition de l'orthographe des mots permettant, pour l'enfant, d'appréhender de façon prédictible la structure graphique des mots.

\subsubsection{Evolution des écritures recensées}

En E1 (avant entraînement), les écritures recensées pour un mot sont très nombreuses. A titre d'exemple, voici un échantillonnage de différentes écritures pour un des mots les plus difficiles, le mot anxiété :

anxieté, ancxiété, anqsiété, encieté, axiété, encsiete, lencieté, anxciété, anxciété, ensiété, ansciété, ancité, anxitée, ensieter, enxité, amxiété, enctieter, anxiétè, enguesiété, insiété, anckiété, anctiété, enqusiété, ennciété, oscécité, onxciété, anxiter, anquecité, lensiètée, ancciété, ansihété, axiéter, exièté, enqisiété, lanqciété, anciété, ancsiété, enciéter, enscité, anxièté, ancièter, anxité, enxiété, ensietté, ensciété, asccitée, enctierté.

Le bi-phonème / ks/ est source de nombreux conflits et, si les écritures proposées témoignent bien de la stratégie phonologique pour le transcrire, elles montrent également la diversité des stratégies, chez les enfants, pour enregistrer à l'écrit / ks/. Elles illustrent le coût cognitif pour les enfants en train d'apprendre l'orthographe : un tel inventaire atteste qu'apprendre l'orthographe lexicale revient à éliminer progressivement tous les possibles pour finalement, « un jour », automatiser la bonne forme graphique du mot. 


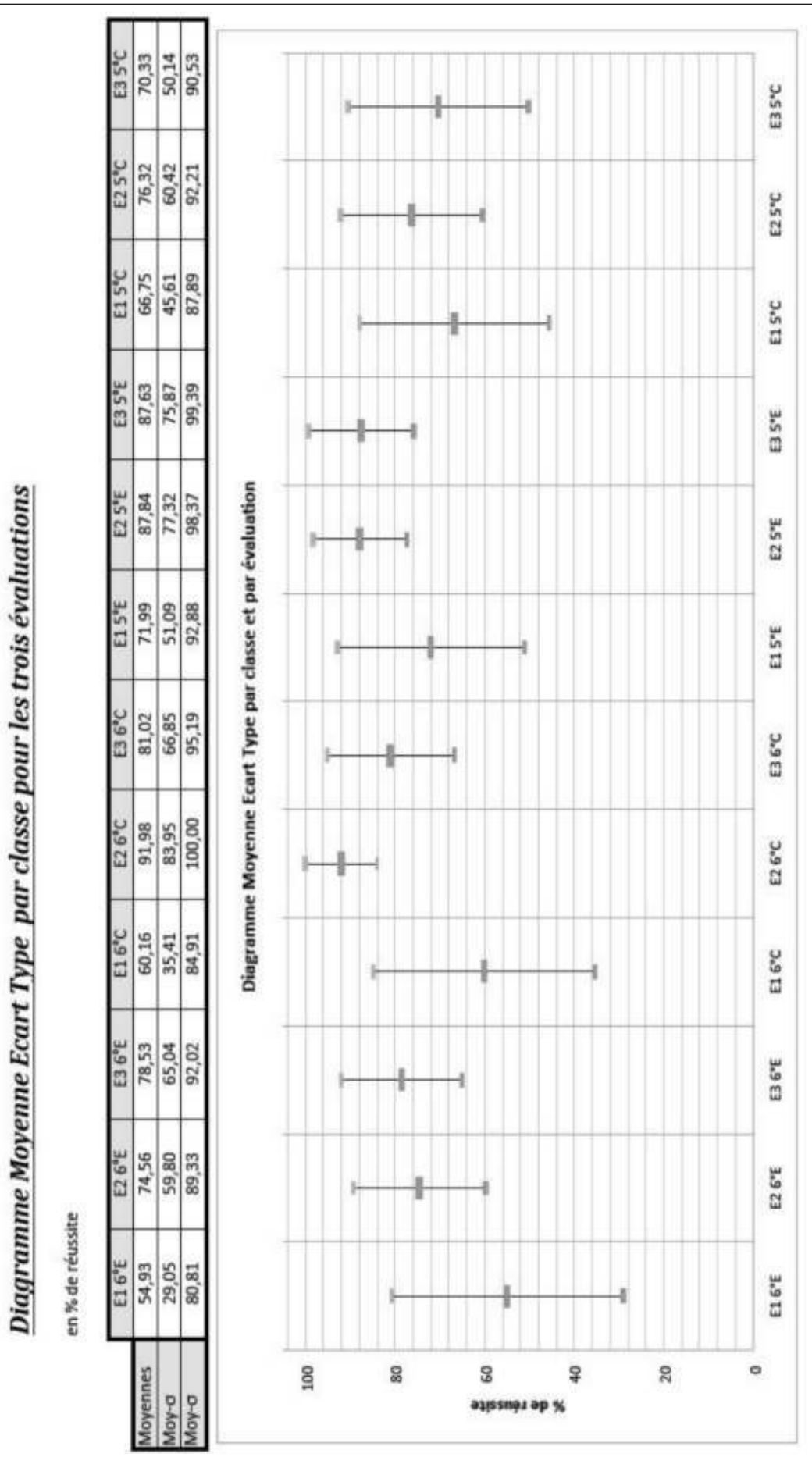

Figure 1 : Moyenne et écart-type par classe (e, entraîné ; c, contrôle), exprimés en pourcentage, aux trois temps de l'évaluation (E1, début, E2, fin, E3, un mois plus tard), en classe de $6^{e}$ et de $5^{e}$. 


\subsubsection{Les mots acquis en E3}

Nous avons extrait les mots acquis par les enfants à $75 \%$ et plus.

\begin{tabular}{|l|c|c|c|c|}
\hline Mots & $6^{\mathrm{e}}$ entraîné & $6^{\mathrm{e}}$ contrôle & $5^{\mathrm{e}}$ entraîné & $5^{\mathrm{e}}$ contrôle \\
\hline Temple & $100 \%$ & $94 \%$ & $100 \%$ & $100 \%$ \\
\hline Conte & $89 \%$ & $82 \%$ & $93 \%$ & $\mathrm{X}$ \\
\hline Béat & $\mathrm{X}$ & $\mathrm{X}$ & $77 \%$ & $\mathrm{X}$ \\
\hline Fan & $85 \%$ & $88 \%$ & $100 \%$ & $79 \%$ \\
\hline Ferveur & $\mathrm{X}$ & $88 \%$ & $91 \%$ & $79 \%$ \\
\hline Orange & $100 \%$ & $100 \%$ & $100 \%$ & $100 \%$ \\
\hline Pudeur & $89 \%$ & $94 \%$ & $95 \%$ & $95 \%$ \\
\hline Strophe & $85 \%$ & $88 \%$ & $93 \%$ & $95 \%$ \\
\hline Pitié & $\mathrm{X}$ & $82 \%$ & $84 \%$ & $79 \%$ \\
\hline Nuisance & $81 \%$ & $\mathrm{X}$ & $81 \%$ & $\mathrm{X}$ \\
\hline Divin & $89 \%$ & $100 \%$ & $93 \%$ & $\mathrm{X}$ \\
\hline Cadence & $\mathrm{X}$ & $\mathrm{X}$ & $88 \%$ & $\mathrm{X}$ \\
\hline Blasphème & $79 \%$ & $76,5 \%$ & $93 \%$ & $\mathrm{X}$ \\
\hline Orgueil & $\mathrm{X}$ & $82 \%$ & $\mathrm{X}$ & $\mathrm{X}$ \\
\hline Las & $81 \%$ & $\mathrm{X}$ & $91 \%$ & $\mathrm{X}$ \\
\hline Destin & $94 \%$ & $100 \%$ & $100 \%$ & $95 \%$ \\
\hline Anxiété & $\mathrm{X}$ & $76,5 \%$ & $84 \%$ & $\mathrm{X}$ \\
\hline Angoisse & $\mathrm{X}$ & $\mathrm{X}$ & $88 \%$ & $\mathrm{X}$ \\
\hline Rite & $77 \%$ & $\mathrm{X}$ & $\mathrm{X}$ & $79 \%$ \\
\hline Bizarre & $83 \%$ & $88 \%$ & $88 \%$ & $\mathrm{X}$ \\
\hline Nerveux & $\mathrm{X}$ & $\mathrm{X}$ & \\
\hline & & & & $\mathrm{X}$ \\
\hline
\end{tabular}

Tableau 2 : Mots acquis en E3 exprimés en pourcentage

En classe de $6^{\mathrm{e}}, 14$ mots sont acquis chez les élèves entraînés et 15 mots, chez les élèves contrôles. En classe de $5^{\mathrm{e}}, 19$ mots sont acquis chez les élèves entraînés et 9 mots, chez les élèves contrôles.

Les élèves contrôles emploient massivement la procédure visuelle pour contrôler l'écriture du mot. Cette procédure semble particulièrement efficace en $6^{\mathrm{e}}$ et moins efficace en $5^{\mathrm{e}}$.

Les élèves entraînés emploient la procédure visuelle et la procédure morphophonologique. Cette dernière semble particulièrement efficace en $5^{\mathrm{e}}$.

\section{Conclusion}

Cette étude a donc mis en évidence l'intérêt de l'entraînement en familles de mots pour la constitution et le maintien d'un lexique orthographique chez des enfants scolarisés en $6^{\mathrm{e}}$ et en $5^{\mathrm{e}}$. L'apprentissage de $1^{\prime}$ 'orthographe lexicale, par l'entraînement morpho-phonologique, permet d'accroître les capacités en orthographe lexicale, capacités qui se maintiendraient après l'entraînement, à la 
différence d'un apprentissage strictement visuel, qui a un impact immédiat mais qui est moins pérenne. Tout comme l'apprentissage de la lecture s'appuie sur deux stratégies (phonologique/sonore et visuelle) modélisées par le modèle à deux voies (phonologique et lexicale), l'apprentissage de l'orthographe lexicale ne se réduirait pas à la voie visuelle mais devrait faire intervenir la voie auditive par l'intégration de la dimension morpho-phonologique. Il y aurait une procédure globale-visuelle et une procédure analytique-morpho-phonologique. La première est plus performante mais ne génère pas, de façon systématique, les correspondances phonème-morphème en graphème. La seconde génère, par un entraînement explicite sur les familles de mots, l'automatisation des correspondances. C'est ainsi que nous pouvons observer une généralisation de la procédure morpho-phonologique en E3 chez les enfants entraînés à l'inverse des enfants contrôles qui ont des résultats plus performants en E2 mais dont on a observé qu'ils ne se maintiennent pas en E3. Nos résultats rejoignent ainsi les travaux de Elbro \& Arnbak (1996), Rieben, et al. (1997), Arnbak \& Elbro (2000), Sabater et al. (2003) et de Pacton (2003).

Par delà le processus cognitif, ces résultats valident également la pertinence de l'écriture prédictible de Doneux (2001). En effet, si les enfants entraînés ont maintenu leur performance un mois après l'entraînement, cela pourrait signifier qu'ils ont découvert une écriture prédictible à partir de leur propre connaissance lexicale et moins de leur mémoire visuelle. Cette manipulation systématique nous semble une piste nouvelle et à poursuivre comme le préconisent déjà Sanguin-Bruckert \& Bruckert (2004).

\section{Références}

ANDERSON, J.R. (2000) : Learning and Memory, New York : Wiley

ARNBAK, E., \& ELBRO, C. (2000) : « The effects of morphological awareness training on the reading and spelling skills of young dyslexics ». Scandinavian Journal of Educational Research, 44 (3), 229-251.

BAUMARD, M. (2004) : «L'école a-t-elle abandonné l'orthographe ? », Le monde de l'Education, 325, 26-29.

Blanche-Benveniste, C., \& Chervel, A. (1969) : L'orthographe. Paris : Maspero.

Bourassa, D. C., \& TREIMAN, R. (2000) : « Children written and oral spelling ». Applied Psycholinguistics, 21, 183-204.

CARlisle, J.F. (1988) : « Knowledge of derivationnal morphology and spelling ability in fourth, sixth, and eighth graders ». Applied psycholinguistics, 9, 247-266 
CATACH, N. (1978) : L'orthographe, Que sais-je? Paris : PUF.

- (1980) : L'orthographe française : Traité théorique et pratique. Paris : Nathan.

COLTHEART, M. (1978) : « Lexical access in simple reading tasks ». In G. Underwood (ed.), Strategies on information processing. London : Academic Press.

DoneuX, J.-L. (2001) : L'écriture du français : Prédictibilité et Aléa, Texte présenté par V. Rey, Aix-en-Provence : PUP.

ELBRO, C., \& ARNBAK, E. (1996) : «The role of morpheme recognition and morphological awareness in dyslexia ». Annals of Dyslexia, 46, 209-240.

FAYOL, M., \& JAFFRÉ, J.-P. (1997) : Orthographes des systèmes aux usages. Paris : Flammarion, coll. Dominos.

Frost, R. \& KATZ, L. (1992) : Orthography, Phonology, Morphology and Meaning, North-Holland : Elsevier Science Publishers.

JAFFRÉ, J.-P. (2003) : L'écriture et les nouvelles technologies : ce que les unes nous apprennent de l'autre. Présenté aux Quatrièmes rencontres Réseaux humains / réseaux technologiques, Université de Poitiers.

LÉté, B., Sprenger-Charolles, L., \& Colé, P. (2004) : « Manulex : A gradelevel lexical database from French elementary-school readers ». Behavior Research Methods, Instruments \& Computers, 36, 156-166.

Morais, J. \& RoBillard, G. (1998) : Apprendre à lire, Paris : Odile Jacob.

PACTON, S. (2003) : «Morphologie et acquisition de l'orthographe : état des recherches actuelles ». Rééducation orthophonique, 213, 27-56.

PothiER, B. (2004) : Echelle d'acquisition en Orthographe Lexicale(EOLE), Paris : Retz.

REY, V. \& GALA, N. (2011) : Les mots de bouche à oreille, En hommage à Alain Giacomi, Paris : Harmattan.

Rieben, L., FAyol, M., \& Perfetti, C. A. (1997) : Des orthographes et leur acquisition. Lausanne : Delachaux \& Nestlé.

Sabater, C., Baudet, V., Habib, M., Prost, V., Thomas, K., \& Rey, V. (2003) : «Déficits phonologiques et morphologiques chez des enfants dyslexiques francophones ». In M.N. Romdhane \& E. Gombert \& M. Belajouza (Eds.), L'apprentissage de la lecture. Perspectives comparatives, pp. 179193. Rennes: Presses Universitaires de Rennes.

SANGUIN-BRUCKERT, C., \& BRUCKERT, J.-P. (2004) : « Le rôle des connaissances morphographiques dans l'acquisition de l'orthographe aux cycles 2 et 3 ». LIDIL, 30, 147-167.

SEYMOUR, P.H.K. (1997) : «Les fondations du développement orthographique et morphographique». In L. Rieben, M. Fayol \& Ch. A. Perfetti (Eds.), Des orthographes et leur acquisition. Paris : Delachaux \& Niestlé, 385-403.

SENECHAL, M. \& KEARNAN, K. (2007) : "The Role of Morphology in reading and spelling ». In R. Kail (Ed.), Advances in Child Development and Behavior, Chap. 8, Vol 35, pp. 297-320, San Diego, CA, US : Elsevier Academic Press.

SPRENGER-ChAROLlES, L., \& CASALIS, S. (1996) : Lire, Lecture et Ecriture : acquisition et troubles du développement. Paris : PUF. 


\section{Annexe 1 : Jeu de mots/ Mots croisés}

Remplir les grilles suivantes en s'aidant des définitions (les définitions sont issues du dictionnaire Le Robert Junior Langue française poche, 2004 et, du dictionnaire Le nouveau Littré, 2006.

\section{Mots croisés : «ANG/ANKS » grille $n^{\circ} 1$}

\section{Définitions :}

1-Très inquiet.

2-Enlever l'inquiétude.

3-Faire éprouver une inquiétude.

4-De manière anxieuse.

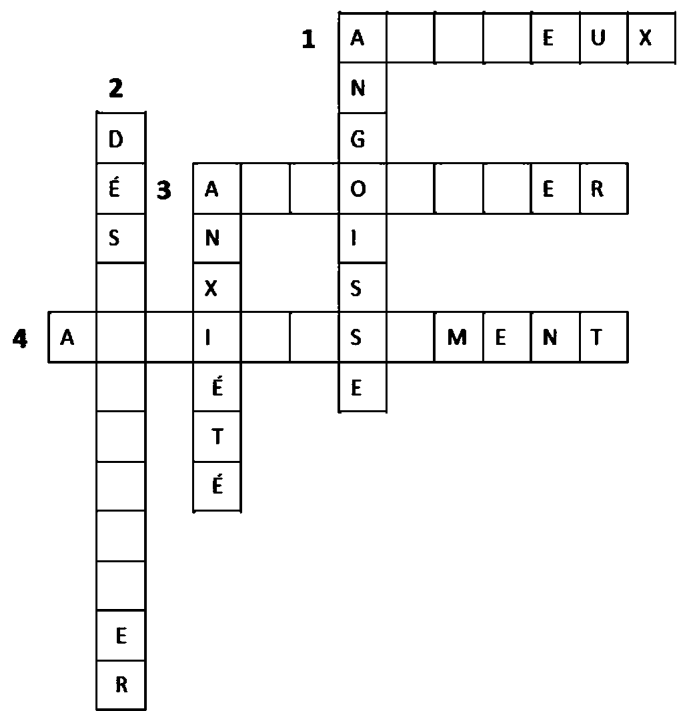

Annexe 2: Mots en escalier

Compléter les cases vierges par les lettres manquantes afin de trouver les bons mots.

Mots en escaliers : Famille 1: ANG/ANKS

\begin{tabular}{|c|c|c|c|c|c|c|c|c|c|c|c|}
\hline \multirow[t]{2}{*}{ A } & $\mathbf{N}$ & $x$ & 1 & É & $\mathbf{T}$ & $\mathbf{E}$ & & & & & \\
\hline & & & & $E$ & $u$ & $x$ & & & & & \\
\hline \multirow[t]{4}{*}{$\mathbf{A}$} & $\mathbf{N}$ & $\mathbf{G}$ & & & & s & E & & & & \\
\hline & & & & & & & $E$ & $\mathbf{R}$ & & & \\
\hline & & & & & & & & M & $E$ & $\mathbf{N}$ & $T$ \\
\hline & & & & & & & & & $S$ & $E$ & $\mathbf{R}$ \\
\hline
\end{tabular}

